University of Wollongong

Research Online

Australian Institute for Innovative Materials -

Papers

Australian Institute for Innovative Materials

$1-1-2014$

\title{
Ambient scalable synthesis of surfactant-free thermoelectric CuAgSe nanoparticles with reversible metallic-n-p conductivity transition
}

Chao Han

University of Wollongong, ch861@uowmail.edu.au

Qiao Sun

University of Wollongong, qsun@uow.edu.au

Zhenxiang Cheng

University of Wollongong, cheng@uow.edu.au

Jianli Wang

University of Wollongong, jianli@uow.edu.au

Zhen Li

University of Wollongong, zhenl@uow.edu.au

See next page for additional authors

Follow this and additional works at: https://ro.uow.edu.au/aiimpapers

Part of the Engineering Commons, and the Physical Sciences and Mathematics Commons

Research Online is the open access institutional repository for the University of Wollongong. For further information contact the UOW Library: research-pubs@uow.edu.au 


\title{
Ambient scalable synthesis of surfactant-free thermoelectric CuAgSe nanoparticles with reversible metallic-n-p conductivity transition
}

\begin{abstract}
Surfactant-free CuAgSe nanoparticles were successfully synthesized on a large scale within a short reaction time via a simple environmentally friendly aqueous approach under room temperature. The nanopowders obtained were consolidated into pellets for investigation of their thermoelectric properties between 3 and $623 \mathrm{~K}$. The pellets show strong metallic characteristics below $60 \mathrm{~K}$ and turn into an n-type semiconductor with increasing temperature, accompanied by changes in the crystal structure (i.e., from the pure tetragonal phase into a mixture of tetragonal and orthorhombic phases), the electrical conductivity, the Seebeck coefficient, and the thermal conductivity, which leads to a figure of merit (ZT) of 0.42 at $323 \mathrm{~K}$. The pellets show further interesting temperature-dependent transition from n-type into $\mathrm{p}$ type in electrical conductivity arising from phase transition (i.e., from the mixture phases into cubic phase), evidenced by the change of the Seebeck coefficient from $-28 \mu \mathrm{V} / \mathrm{K}$ into $226 \mu \mathrm{V} / \mathrm{K}$ at $467 \mathrm{~K}$. The ZT value increased with increasing temperature after the phase transition and reached 0.9 at $623 \mathrm{~K}$. The sintered CuAgSe pellets also display excellent stability, and there is no obvious change observed after 5 cycles of consecutive measurements. Our results demonstrate the potential of CuAgSe to simultaneously serve (at different temperatures) as both an n-type and a p-type thermoelectric material.
\end{abstract}

\section{Keywords}

surfactant, free, thermoelectric, cuagse, nanoparticles, synthesis, reversible, ambient, metallic, $n, p$, conductivity, transition, scalable

\section{Disciplines}

Engineering | Physical Sciences and Mathematics

\section{Publication Details}

Han, C., Sun, Q., Cheng, Z. Xiang., Wang, J. Li., Li, Z., Lu, G. \& Dou, S. Xue. (2014). Ambient scalable synthesis of surfactant-free thermoelectric CuAgSe nanoparticles with reversible metallic-n-p conductivity transition. Journal of the American Chemical Society, 136 (50), 17626-17633.

\section{Authors}

Chao Han, Qiao Sun, Zhenxiang Cheng, Jianli Wang, Zhen Li, Gao Qing (Max) Lu, and S X. Dou 


\title{
Ambient Scalable Synthesis of Surfactant-free Thermoelectric CuAgSe Nanoparticles with Reversible Metallic-n-p Conduc- tivity Transition
}

\author{
Chao $\operatorname{Han}^{\dagger}$, Qiao Sun ${ }^{\dagger, \ddagger}$, Zhen Xiang Cheng ${ }^{\dagger}$, Jian Li Wang ${ }^{\dagger}$, Zhen Li*†, ${ }^{\dagger}$, Gao Qing (Max) Lu ${ }^{\S}$, Shi Xue \\ Dou $^{\dagger}$
}

†Institute for Superconducting and Electronic Materials, Australian Institute for Innovative Materials, University of Wollongong, Squires Way, North Wollongong, NSW 2500, Australia.

$\ddagger$ \$chool of Radiation Medicine and Radiation Protection, Soochow University, 199 Ren Ai Road, Suzhou Industrial Park, Suzhou 215123, China.

§Australian Institute for Bioengineering \& Nanotechnology, The University of Queensland, Brisbane, QLD, 4072, Australia

\section{Supporting Information Placeholder}

\begin{abstract}
Surfactant-free CuAgSe nanoparticles were successfully synthesized on a large scale within a short reaction time via a simple environmentally friendly aqueous approach under room temperature. The nanopowders obtained were consolidated into pellets for investigation of their thermoelectric properties between $3 \mathrm{~K}$ and $623 \mathrm{~K}$. The pellets show strong metallic characteristics below $60 \mathrm{~K}$ and turn into an $n$-type semiconductor with increasing temperature, accompanied by changes in the crystal structure (i.e. from the pure tetragonal phase into a mixture of tetragonal and orthorhombic phases), the electrical conductivity, the Seebeck coefficient, and the thermal conductivity, which leads to a figure of merit (ZT) of 0.42 at $323 \mathrm{~K}$. The pellets show further interesting temperature-dependent transition from $n$-type into $p$-type in electrical conductivity arising from phase transition (i.e. from the mixture phases into cubic phase), evidenced by the change of the Seebeck coefficient from -28 $\mu \mathrm{V} / \mathrm{K}$ into $226 \mu \mathrm{V} / \mathrm{K}$ at $467 \mathrm{~K}$. The $Z T$ value increased with increasing temperature after the phase transition and reached 0.9 at $623 \mathrm{~K}$. The sintered CuAgSe pellets also display excellent stability, and there is no obvious change observed after 5 cycles of consecutive measurements. Our results demonstrate the potential of $\mathrm{CuAgSe}$ to simultaneously serve (at different temperatures) as both an $n$-type and a $p$ type thermoelectric material.
\end{abstract}

\section{INTRODUCTION}

Direct conversion of heat into electricity on the basis of the Seebeck effect (i.e. thermoelectric technology, TE) shows great potential in harvesting waste heat, because it is environmentally friendly, stable, reliable, and applicable in remote areas. ${ }^{1-4}$ This technology could lead to significant savings on energy consumption and reduce carbon emissions. It remains a challenge, however, for wide practical applications because of its low conversion efficiency arising from the lack of environmentally friendly, high performance, and low cost thermoelectric materials. The thermoelectric performance of materials is characterized by the "figure of merit" $(Z T)$, which is described by Equation (1).

$$
Z T=\frac{S^{2} \sigma}{\kappa} T
$$

In which $S$ is the Seebeck coefficient, $\sigma$ is the electrical conductivity, $\kappa$ is the thermal conductivity, and $S^{2} \sigma$ is defined as the power factor, while $T$ is the measurement temperature. $^{5-11}$

Great efforts have been devoted to optimization of these 3 factors for achieving a high ZT. It is very challenging, however, to simultaneously optimize them; an alternative way is the introduction of nanostructures or the use of phononglass electron-crystal (PGEC) or phonon-liquid electroncrystal (PLEC) materials to reduce the thermal conductivity (while the power factor is not seriously degraded) to achieve a high $Z T .^{1-8,12-13}$ For example, the $Z T$ value of $p$-type PbTe can be improved to over 2 by the introduction of nanoprecipitates through chemical doping. ${ }^{14}$ The concept of PGEC was proposed by Slack et al in 1979, and it has attracted considerable attention in the regime of thermoelectric materials. On the basis of PGEC, a similar concept PLEC was proposed in 2012 during investigation of thermoelectric properties of non-stoichiometric superionic copper selenide $\left(\mathrm{Cu}_{2-x} \mathrm{Se}\right)$, which exhibits a $Z T$ of 1.6 at $1000 \mathrm{~K}$ due to the phase change from rigid crystal to disordered PLEC state, which was composed by highly disordered cuprous cations around with the chalcogen sublattice. The copper ions behave like a liquid at high temperature and minimize the thermal conductivity. ${ }^{12}$ Another good example of PLEC material is nonstoichiometric copper sulfide $\mathrm{Cu}_{1.97} \mathrm{~S}$, which has the maximum $Z T$ of 1.7 at $1000 \mathrm{~K}$ accompanied with an obvious phase transition from low chalcocite phase to PLEC state at around 
$400 \mathrm{~K} .{ }^{15}$ Besides copper chalcogenides, silver chalcogenides experienced similar phase transition and anomalous TE performance was also observed. ${ }^{16}$ Although the phase transition related transports are common in PLEC materials, its mechanism was rarely studied..$^{12,15-17}$

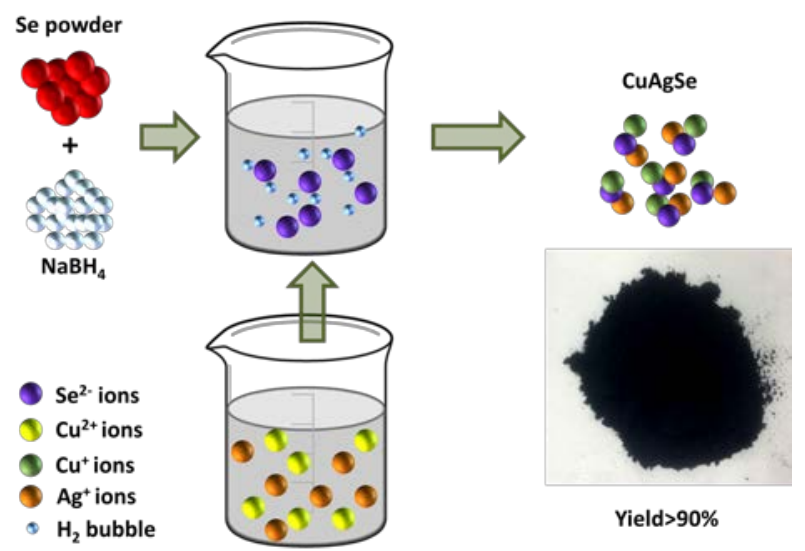

Scheme 1. Schematic illustration of synthesis process.

The high ZT of the PLEC has motivated substantial efforts in searching for high performance thermoelectric materials with a similar superionic structure. As an important ternary selenide, $\mathrm{CuAgSe}$ is such a candidate with the superionic property due to the mobility of both $\mathrm{Cu}^{+}$and $\mathrm{Ag}^{+}$ions at high temperature. Extremely high electron mobility at low temperature and a $Z T$ of 0.15 at room temperature and a $Z T$ of 0.95 at $623 \mathrm{~K}$ have been reported in bulk CuAgSe. ${ }^{18-19}$

It should be noted that most research has been focused on bulk $\mathrm{CuAgSe}$ prepared by the solid state reaction at high temperature $(>1000 \mathrm{~K}),{ }^{18-19}$ and there is no report on the thermoelectric properties of $\mathrm{CuAgSe}$ sintered from its nanostructures due to the difficulties in efficient preparation of $\mathrm{CuAgSe}$ nanomaterials on a large scale. Small-scale $\mathrm{CuAgSe}$ nanotubes and dendrites can be prepared from Se and $\mathrm{Ag}_{2} \mathrm{Se}$ nanotemplates, respectively. ${ }^{20-21}$ Herein, we synthesize surfactant-free $\mathrm{CuAgSe}$ nanoparticles from $\mathrm{Cu}\left(\mathrm{NO}_{3}\right)_{2}$, $\mathrm{AgNO}_{3}$, and Se precursors via a robust ambient approach (Scheme 1). The whole synthesis process is very simple and effective (within $30 \mathrm{~min}$ ) with a high yield (over 90\%). More importantly, the whole reaction is in an aqueous system without heating or any expensive/toxic solvents, and dangerous waste. This simple and scalable method is capable of producing large amounts of surfactant-free CuAgSe nanoparticles for investigation of the thermoelectric properties of their sintered pallets between $3 \mathrm{~K}$ and $623 \mathrm{~K}$. The results show their excellent electric conductivity at low temperature (below 6o K) and semiconducting characteristics at high temperature, which can be reversibly switched from metallic conductor, $n$-type semiconductor to $p$-type semiconductor by varying the temperature. The $Z T$ values of $\mathrm{CuAgSe}$ made from nanoparticles are 0.42 at $323 \mathrm{~K}$ and 0.9 at $623 \mathrm{~K}$, respectively.

\section{EXPERIMENTAL SECTION}

\subsection{Synthesis of CuAgSe nanoparticles}

All chemicals were bought from Sigma-Aldrich Pty. Ltd. and used as received without any further purification. In a typical synthesis, $3.16 \mathrm{~g}$ (40 mmol) Se powder (100 mesh, $\geq 99.5 \%$ ) and $4.54 \mathrm{~g}$ (120 mmol) $\mathrm{NaBH}_{4}$ (caplets, $\geq 98 \%$ ) were dispersed in $400 \mathrm{~mL}$ distilled water, and the mixture was stirred for 25 min under the protection of $\mathrm{Ar}$ at room temperature to form a colorless selenium precursor solution. $9.7 \mathrm{~g}$ (40 mmol) $\mathrm{Cu}\left(\mathrm{NO}_{3}\right)_{2} \cdot 3 \mathrm{H}_{2} \mathrm{O}(97 \%)$ and $6.8 \mathrm{~g}$ (40 mmol) $\mathrm{AgNO}_{3}$ (97\%) were completely dissolved in $400 \mathrm{~mL}$ distilled water, and then quickly added into the Se-precursor solution to generate black precipitates. The black product was washed with distilled water several times, and then dried to a constant weight at $80{ }^{\circ} \mathrm{C}$ in a vacuum to give a yield over $90 \%$.

\subsection{Characterization}

$\mathrm{X}$-ray diffraction (XRD) studies were carried out at room temperature with an X-ray diffractometer (GBC-MMA) using $\mathrm{Cu}-\mathrm{K}_{\alpha}$ radiation $(\lambda=0.154 \mathrm{~nm})$. The sample was characterized by X-ray powder diffraction (XRD) measurements in the temperature range between $20 \mathrm{~K}$ and $300 \mathrm{~K}$ using a PANalytical diffractometer with $\mathrm{Cu}-\mathrm{K}_{\alpha}$ radiation $(\lambda=0.154$ $\mathrm{nm})$. Scanning electron microscopy (SEM) was conducted on a JEOL JSM-75ooFA microscope. Transmission electron microscopy (TEM) was performed using a JEOL JEM-2011 microscope with an accelerating voltage of $200 \mathrm{kV}$. X-ray photoelectron spectroscopy (XPS) was conducted using a SPECS PHOIBOS 100 Analyzer installed in a high-vacuum chamber with the base pressure below $10^{-8}$ mbar. X-ray excitation was provided by $\mathrm{Al} \mathrm{K}_{\alpha}$ radiation with the photon energy $h v=$ $1486.6 \mathrm{eV}$ at the high voltage of $12 \mathrm{kV}$ and power of $120 \mathrm{~W}$. The binding energy spectra were recorded at the pass energy of $20 \mathrm{eV}$ in the fixed analyzer transmission mode. All the spectra were calibrated with $\mathrm{C}$ is at $284.6 \mathrm{eV}$. Analysis of the XPS data was carried out using the commercial Casa XPS 2.3.15 software package.

\subsection{Thermoelectric properties test}

The as-synthesized CuAgSe nanoparticles ( $3 \mathrm{~g}$ ) were loaded into a graphite die with a diameter of $20 \mathrm{~mm}$ and sintered into a pellet at $430{ }^{\circ} \mathrm{C}$ under $65 \mathrm{MPa}$ pressure for $10 \mathrm{~min}$ by the spark plasma sintering (SPS) technique. The pellet was cut into rectangular pieces with dimensions of $4 \times 13 \times 1.2 \mathrm{~mm}^{3}$ for thermoelectric characterization.

Low-temperature thermoelectric performance (i.e. from 3 $\mathrm{K}$ to $350 \mathrm{~K}$ ) was tested on a physical properties measurement system (PPMS). The Seebeck coefficient, electrical conductivity, and thermal conductivity, as well as the $Z T$ values, were obtained simultaneously. The high-temperature Seebeck coefficient and electrical conductivity were measured from $323 \mathrm{~K}$ to $623 \mathrm{~K}$ under helium atmosphere using an Ozawa RZ2ooi (Japan) instrument. A Linseis LFA1ooo (Germany) was used to determine the thermal diffusivity of samples, which were cut and polished into $\varnothing_{10 \times 1} \mathrm{~mm}^{2}$ disks. The thermal conductivity $(\kappa)$ was calculated using the equation $\kappa$ $=D \times C_{\mathrm{p}} \times \rho$, where $D$ is the thermal diffusivity, $C_{\mathrm{p}}$ is the heat capacity, and $\rho$ is the mass density of the specimen, determined by the Archimedes method. The temperature dependence of the heat capacity $\left(C_{\mathrm{p}}\right)$ was determined using the differential scanning calorimeter (DSC) method on a DSCQ1oo 
instrument with a heating rate of $10 \mathrm{~K} / \mathrm{min}$ and a $\mathrm{N}_{2}$ flow of $50 \mathrm{~mL} / \mathrm{min}$.

\subsection{Theoretical calculations}

The calculations of band structures of tetragonal and cubic $\alpha$-phase $\mathrm{CuAgSe}$ were performed using the generalized gradient approximation ${ }^{22}$ treated by the Perdew-Burke-Ernzerh (PBE) of exchange-correlation potential, as implemented in the $\mathrm{DMol}_{3}$ module in Materials Studio. ${ }^{23-24}$ An all electrons double numerical atomic orbital augmented by $\mathrm{d}$ polarization functions (DNP) was used as basis set, which has been successfully used to determine the electronic structures of nanomaterials. ${ }^{25}$ The calculation models were built according to the experimental lattice parameters. ${ }^{26-27}$ The electronic Brillouin zone was sampled by using a MonkhorstPack grid of sample spacing $0.002 \AA^{-1}$.

\section{RESULTS AND DISCUSSION}

It is well known that $\mathrm{CuAgSe}$ has tetragonal, orthorhombic, and cubic structures (Figure 1), in which the tetragonal structure has lattice parameters $a=4.1 \AA, b=4.05 \AA$, and $c=6.31$ $\AA$, and the orthorhombic one is an essentially a pseudotetragonal structure with a large supercell $(a=4.1 \AA, b=$ $20.35 \AA$, and $c=6.31 \AA) .{ }^{26,28}$ The "tetragonal structure" here is not strictly tetragonal as $a \neq b(a=4.1 \AA$ and $b=4.05$ (a)

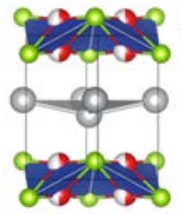

(b) 0

(c)

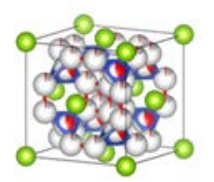

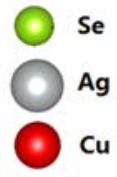

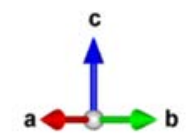

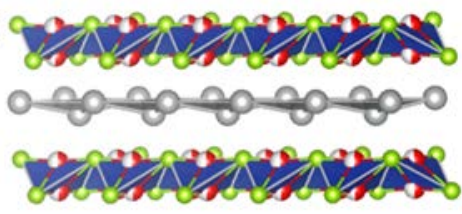

Figure 1. Schematic crystal structures of CuAgSe: (a) low temperature tetragonal CuAgSe; (b) low temperature orthorhombic CuAgSe; (c) high temperature cubic CuAgSe. The mixed gray and red mean randomly occupied by Ag or $\mathrm{Cu}$ atoms.

$\AA$ ). There is no big difference between these two structures, except that the orthorhombic structure features a sequence which repeats itself periodically every five tetragonal cells in the $b$ direction. They both possess a similar layered structure consisting of alternating stacking of the $\mathrm{Ag}$ and CuSe layers, in which $\mathrm{Ag}$ atoms almost lie in the same plane and are bonded closely to Se atoms, allowing high mobility of $\mathrm{Ag}$ atoms and the formation of Ag-Ag metallic bonds. ${ }^{26}$ Se atoms form a squashed tetrahedron, in which each corner is shared with adjacent tetrahedron, and $\mathrm{Cu}$ atoms are offset from the center within the tetrahedron. Each tetragonal cell contains two CuAgSe molecules, and the orthorhombic one has ten molecules. Both the tetragonal and the orthorhombic structures are stable at low temperature and can be reversibly transformed into the cubic structure at high temperature.
The crystal structure of the as-synthesized CuAgSe nanoparticles was determined by X-ray diffraction, and their XRD pattern is compared with those of standard orthorhombic CuAgSe (JCPDS 10-0451, black vertical lines) and tetragonal CuAgSe (JCPDS 25-1180, red vertical lines) in Figure 2(a). Most peaks and their intensity ratios are well matched with those of the orthorhombic structure, except for the peaks at $2 \theta=43.16^{\circ}(0,0,3), 44.02^{\circ}(2,0,0)$, and $44.5^{\circ}(0,2,0)$, which are marked with black dots in Figure 2(a) and can be solely attributed to the tetragonal structure. The mixture of tetragonal and orthorhombic structures is attributed to the minor difference between them, and this could be caused by the nanoscale effects and the distortion of the crystal during the formation of nanoparticles.

The SEM and TEM images in Figure 2(b-c) show irregular CuAgSe nanoparticles with an average diameter of $20 \mathrm{~nm}$. The high resolution TEM (HRTEM) image in Figure 2(d) clearly shows an interplanar spacing of $0.155 \mathrm{~nm}$, which is consistent with the $(1,10,2)$ plane. The fast Fourier transform (FFT) image in the inset of Figure 2 (d) shows the diffraction spots from the $(0,10,0)$ and $(1,6,0)$ crystal planes.

The resultant $\mathrm{CuAgSe}$ nanoparticles were further characterized by XPS, and Figure 3 shows the binding energies of elements in the sample after calibration with the binding energy of $\mathrm{C}$ is at $284.6 \mathrm{eV}$. The absence of other elements apart from $\mathrm{C}, \mathrm{O}, \mathrm{Cu}, \mathrm{Ag}$, and Se indicates the high purity of
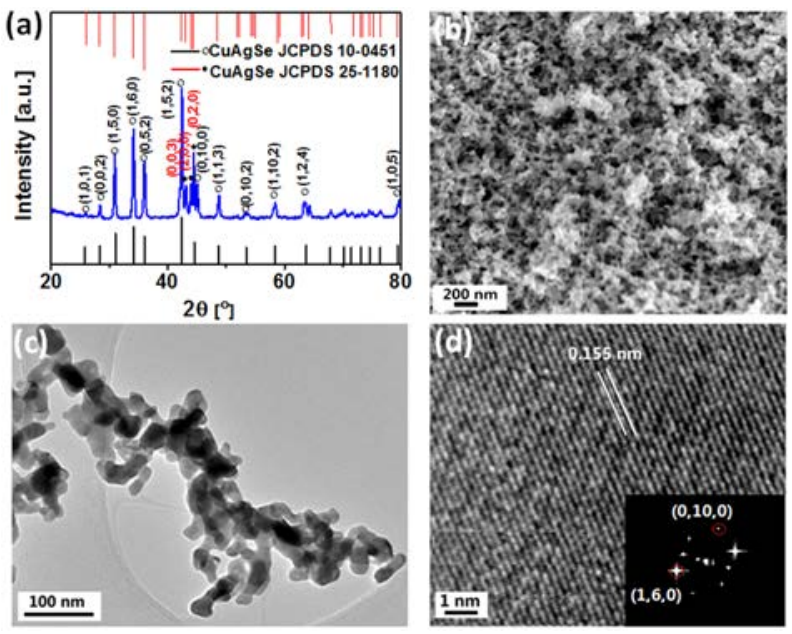

Figure 2. (a) X-Ray diffraction pattern, (b) SEM image of the prepared nanoparticles; (c) TEM and (d) HRTEM images of as-synthesized $\mathrm{CuAgSe}$ nanoparticles, inset picture is Fast Fourier Transform pattern from HRTEM image.

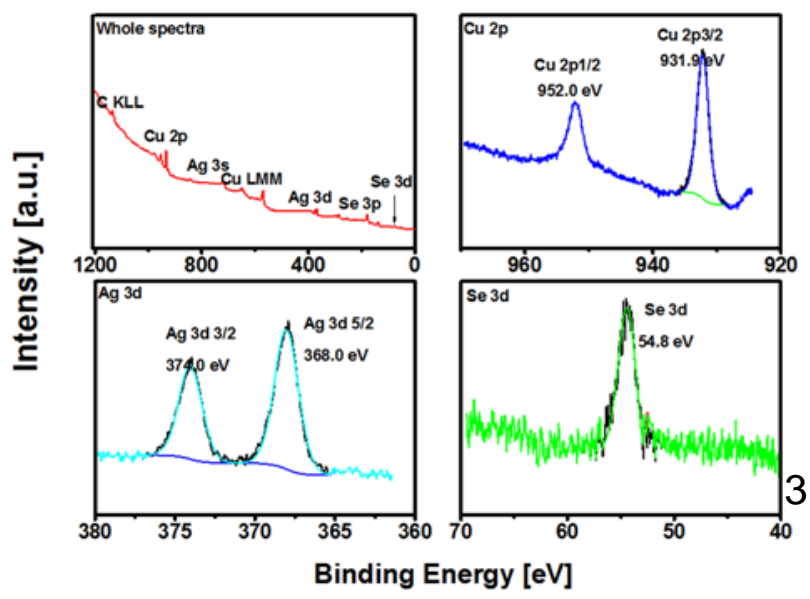

Figure 3. XPS spectra of the CuAgSe nanopowder. 
the nanoparticles. The two distinct peaks at $931.9 \mathrm{eV}$ and 952.0 eV in the $\mathrm{Cu} 2 \mathrm{p}$ spectrum are assigned to $\mathrm{Cu} 2 \mathrm{p} 3 / 2$ and $\mathrm{Cu} 2 \mathrm{p} 1 / 2$ of $\mathrm{Cu}^{+}$, respectively. The absence of a satellite peak at $\sim 942.47 \mathrm{eV}$ further supports the identification of the copper element as $\mathrm{Cu}^{+}$, rather than $\mathrm{Cu}^{2+}{ }^{29}$ The peaks located at 368.0 and $374.0 \mathrm{eV}$ represent the $3 \mathrm{~d}_{5} / 2$ and $3 \mathrm{~d}_{3} / 2$ orbital of $\mathrm{Ag}^{+}$, respectively. The Se $3 \mathrm{~d}_{5} / 2$ peak from $\mathrm{Se}^{2-}$ is located at $54.8 \mathrm{eV}^{11,30}$ The binding energies of $\mathrm{Cu}^{+}, \mathrm{Ag}^{+}$, and $\mathrm{Se}^{2-}$ ions are consistent with previous reports, demonstrating the formation of pure $\mathrm{CuAgSe}$ nanoparticles. ${ }^{20,31-33}$

The above results demonstrate that pure $\mathrm{CuAgSe}$ nanoparticles can be simply synthesized from $\mathrm{Cu}\left(\mathrm{NO}_{3}\right)_{2}, \mathrm{AgNO}_{3}$, and Se precursors in aqueous solution. The formation mechanism is not as simple as Scheme 1 shown, however, and includes the following key steps. Firstly, selenium powder is reduced by $\mathrm{NaBH}_{4}$ to form the colorless Se-precursor solution, as shown in Reaction (2).

$2 \mathrm{Se}+4 \mathrm{NaBH}_{4}+7 \mathrm{H}_{2} \mathrm{O} \rightarrow 2 \mathrm{NaHSe}+\mathrm{Na}_{2} \mathrm{~B}_{4} \mathrm{O}_{7}+14 \mathrm{H}_{2} \uparrow \quad$ (2)

After addition of a mixture of $\mathrm{Cu}\left(\mathrm{NO}_{3}\right)_{2}$ and $\mathrm{AgNO}_{3}$ into the Se-precursor solution, the excess $\mathrm{NaBH}_{4}$ in-situ reduces $\mathrm{Cu}^{2+}$ into $\mathrm{Cu}^{+}$through Reaction (3) because the standard reduction potential of $\mathrm{Cu}^{2+} / \mathrm{Cu}^{+}(0.153 \mathrm{~V})$ is much smaller than that of $\mathrm{Ag}^{+} / \mathrm{Ag}(0.8 \mathrm{~V})$.

$2 \mathrm{NaBH}_{4}+2 \mathrm{Cu}^{2+}+4 \mathrm{H}_{2} \mathrm{O} \rightarrow 2 \mathrm{Cu}^{+}+2 \mathrm{H}^{+}+7 \mathrm{H}_{2} \uparrow+2 \mathrm{NaBO}_{2}$ (3)

(a)
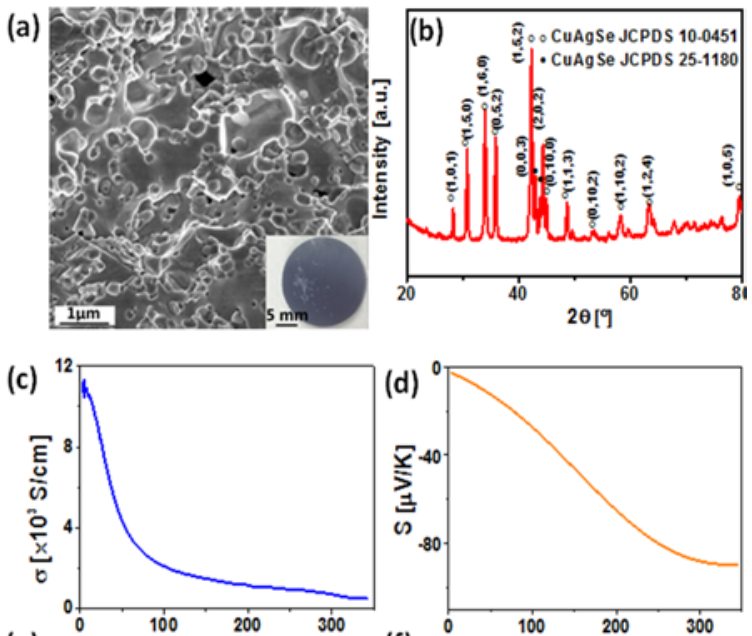

(e)

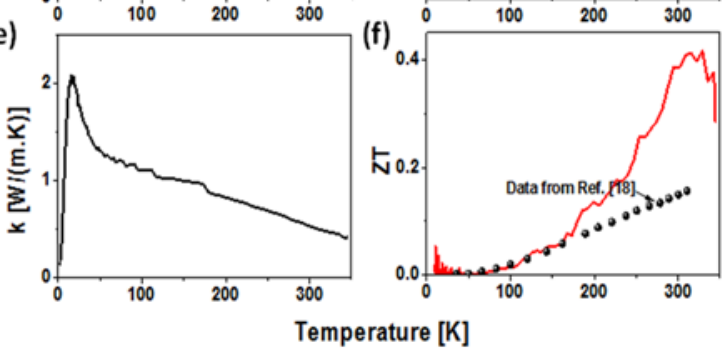

Figure 4. (a) SEM image of the consolidated pellet from the prepared $\mathrm{CuAgSe}$ nanoparticles, inset is the coin-like pellet; (b) XRD pattern of the pellet; (c-f) TE performance of the sintered $\mathrm{CuAgSe}$ nanoparticles from $3 \mathrm{~K}$ to room

Then, $\mathrm{AgNO}_{3}$ reacts with NaHSe to form $\mathrm{Ag}_{2}$ Se precipitate first, due to the lower solubility of $\mathrm{Ag}_{2} \mathrm{Se}$ compared to $\mathrm{Cu}_{2} \mathrm{Se}$ $\left[K_{\mathrm{sp}}\left(\mathrm{Ag}_{2} \mathrm{Se}\right)=2 \times 10^{-63.7} ; K_{\mathrm{sp}}\left(\mathrm{Cu}_{2} \mathrm{Se}\right)=1.58 \times 10^{-60.8}\right]$, followed by the reaction of fresh $\mathrm{Ag}_{2} \mathrm{Se}$ with $\mathrm{Cu}_{2} \mathrm{Se}$ to form alloyed $\mathrm{CuAgSe}$ nanoparticles through Reaction (4). ${ }^{20-21}$

$$
\mathrm{Ag}_{2} \mathrm{Se}+\mathrm{Cu}_{2} \mathrm{Se} \rightarrow 2 \mathrm{CuAgSe} \text { (4) }
$$

To prove the above mechanism, a control experiment was conducted by using less reducing agent (i.e. $\mathrm{Se} / \mathrm{NaBH}_{4}$ molar ratio of 1:2) and using $\mathrm{CuCl}$ to replace $\mathrm{Cu}\left(\mathrm{NO}_{3}\right)_{2}$. After the Se powder was completely reduced by $\mathrm{NaBH}_{4}$ to form a clear solution, the $\mathrm{AgNO}_{3}$ solution was added into the Seprecursor solution to form a black suspension. A portion of the suspension was separated from solution and purified for analysis. $\mathrm{CuCl}$ powder was dissolved in $\mathrm{HCl}$ solution and added into black suspension under stirring. The mixture was stirred for $30 \mathrm{~min}$ at room temperature, and then the precipitate was collected. The XRD patterns and morphology of the black precipitates collected before and after addition of $\mathrm{CuCl}$ solution are shown in Figure $S_{1}$ in the Supporting Information. The results demonstrate that the precipitate collected before the addition of $\mathrm{CuCl}$ is pure $\mathrm{Ag}_{2} \mathrm{Se}$ (JCPDS 24-1041), and the one collected after addition of $\mathrm{CuCl}$ is $\mathrm{CuAgSe}$ mixed with a small amount of $\mathrm{Ag}_{2} \mathrm{Se}$. These results demonstrate that residual $\mathrm{Se}^{2-}$ ions react with $\mathrm{Cu}^{+}$to form $\mathrm{Cu}_{2} \mathrm{Se}$, which further reacts with $\mathrm{Ag}_{2} \mathrm{Se}$ to form $\mathrm{CuAgSe}$. This mechanism is further supported by the reaction between the as-prepared $\mathrm{Cu}_{2} \mathrm{Se}$ nanoparticles and the $\mathrm{Ag}_{2} \mathrm{Se}$ nanoparticles. As displayed in Figure $S_{2}$, the typical diffraction peak $(1,6,0)$ from orthorhombic $\mathrm{CuAgSe}$ was observed in the mixture after it was stirred for $10 \mathrm{~min}$ at room temperature. The intensity of this peak was increased in the mixture after $30 \mathrm{~h}$ aging time under stirring, indicating the increased amount of $\mathrm{CuAgSe}$ nanoparticles in the mixture, although the $\mathrm{Cu}_{2} \mathrm{Se}$ and $\mathrm{Ag}_{2} \mathrm{Se}$ nanoparticles were not completely transformed into $\mathrm{CuAgSe}$.

According to the reaction mechanism, the compositions of the final products strongly depend on the precursor ratio of $\mathrm{Cu}^{2+}: \mathrm{Ag}^{+}: \mathrm{Se}^{2-}\left(R\right.$, molar ratio) and the amount of $\mathrm{NaBH}_{4}$. As shown in Figure $\mathrm{S}_{3}(\mathrm{a})$, CuAgSe nanoparticles can only be obtained with a ratio of 1:1:1. When a ratio of 1:2:2 was used, the product is a mixture of $\mathrm{CuAgSe}, \mathrm{Ag}_{2} \mathrm{Se}$ (JCPDS 24-1041), and Ag (JCPDS 65-2871) nanoparticles, as indicated by the $\mathrm{XRD}$ pattern. In the case of 2:2:1 ratio, the product is a mixture of $\mathrm{Ag}_{2} \mathrm{Se}$ and $\mathrm{Ag}$ nanoparticles. The appearance of $\mathrm{Ag}$ in both cases could be due to the reduction of $\mathrm{Ag}^{+}$by slightly excess $\mathrm{NaBH}_{4}$. When a 2:1:2 ratio was adopted, the product contained CuAgSe and a small amount of $\mathrm{Cu}_{2} \mathrm{Se}$ (JCPDS 652982).

The role of $\mathrm{NaBH}_{4}$ is to reduce both the Se powder and the $\mathrm{Cu}\left(\mathrm{NO}_{3}\right)_{2}$, and it is crucial to control the amount of $\mathrm{NaBH}_{4}$. The ideal amount of $\mathrm{NaBH}_{4}$ should be 3 times that of $\mathrm{Cu}\left(\mathrm{NO}_{3}\right)_{2}$ (or $\mathrm{AgNO}_{3}$ or Se), i.e. their molar ratio $r$ should be 3:1. When an inadequate amount of $\mathrm{NaBH}_{4}$ was used (i.e. $r=$ 2:1), the product is a mixture of $\mathrm{Ag}_{2} \mathrm{Se}, \mathrm{CuSe}$, and $\mathrm{Se}$, as shown in Figure $\mathrm{S}_{3}(\mathrm{~b})$, because $\mathrm{NaBH}_{4}$ is not enough for reduction of the Se powder and $\mathrm{Cu}\left(\mathrm{NO}_{3}\right)_{2}$. When excessive $\mathrm{NaBH}_{4}$ (e.g. $r=4: 1$ or 8:1) was used, $\mathrm{Ag}$ and $\mathrm{Cu}_{2} \mathrm{O}$ were observed in the products [Figure $\mathrm{S}_{3}(\mathrm{~b})$ ]. The formation of $\mathrm{Cu}_{2} \mathrm{O}$ could be due to the increased solution $\mathrm{pH}$ caused by the hydrolysis of $\mathrm{NaBH}_{4}$ and sodium borate.

This simple green method is capable of producing $\mathrm{CuAgSe}$ nanoparticles on the gram scale in a one-pot reaction. The resultant surfactant-free nanoparticles can be directly used without surface modification for fabrication of pellets for thermoelectric study. Figure 4(a) presents an SEM image of 
the cross-section of a pellet [the inset in Figure 4(a)], which was made from as-synthesized $\mathrm{CuAgSe}$ nanoparticles that were consolidated at $430{ }^{\circ} \mathrm{C}$ for 10 min under $65 \mathrm{MPa}$ using the SPS technique. The relative density is about $90 \%$, and the SEM image shows nanoscale pores- and particles in the pellet, although most nanoparticles were coarsened and aggregated to form larger grains. Compared with non-sintered powder, there is no distinct difference in its roomtemperature crystal structure [Figure 4(b)], except that the diffraction intensity of the crystal planes [e.g. $(0,0,3)$ peak] of the tetragonal phase is improved.

The pellet was cut into pieces for thermoelectric investigation, and Figure 4(c)-(f) presents the results obtained on a PPMS instrument from low temperature to room temperature. The temperature dependence of the electrical conductivity in Figure 4(c) clearly demonstrates the metallic behavior of the pellet below $60 \mathrm{~K}$, as indicated by the gradual increase of the electrical conductivity from $800 \mathrm{~S} / \mathrm{cm}$ at room temperature to $3500 \mathrm{~S} / \mathrm{cm}$ at $60 \mathrm{~K}$, followed by a rapid increase to $11330 \mathrm{~S} / \mathrm{cm}$ at $3 \mathrm{~K}$. The tendency of the Seebeck coefficient is opposite to that of the electrical conductivity, and it decreases from $-90 \mu \mathrm{V} / \mathrm{K}$ to nearly o with temperature decreasing from room temperature to $3 \mathrm{~K}$ [Figure $4(\mathrm{~d})$ ].

The variation of the thermal conductivity with temperature is shown in Figure 4(e). It rises with temperature decreasing to reach the maximum value of $2.1 \mathrm{~W} /(\mathrm{m} \cdot \mathrm{K})$ at $22 \mathrm{~K}$, and then decreases to $0.2 \mathrm{~W} /(\mathrm{m} . \mathrm{K})$ at $3 \mathrm{~K}$. The ultra-low thermal conductivity $(0.5 \mathrm{~W} /(\mathrm{m} \cdot \mathrm{K}))$ at room temperature can be attributed to the large lattice anharmonicity caused by the structure, which is reminiscent of amorphous solids. ${ }^{18}$ In addition, the nanoscale particles, pores, and grain boundaries that are present in the pellet also make contributions to the low thermal conductivity, because they can effectively scatter different wavelengths of phonons. The $Z T$ value of the pellet is displayed in Figure $4(\mathrm{f})$, and it gradually increases with increasing temperature to achieve a value of 0.42 at 323 $\mathrm{K}$, and then decreases with temperature. Compared with

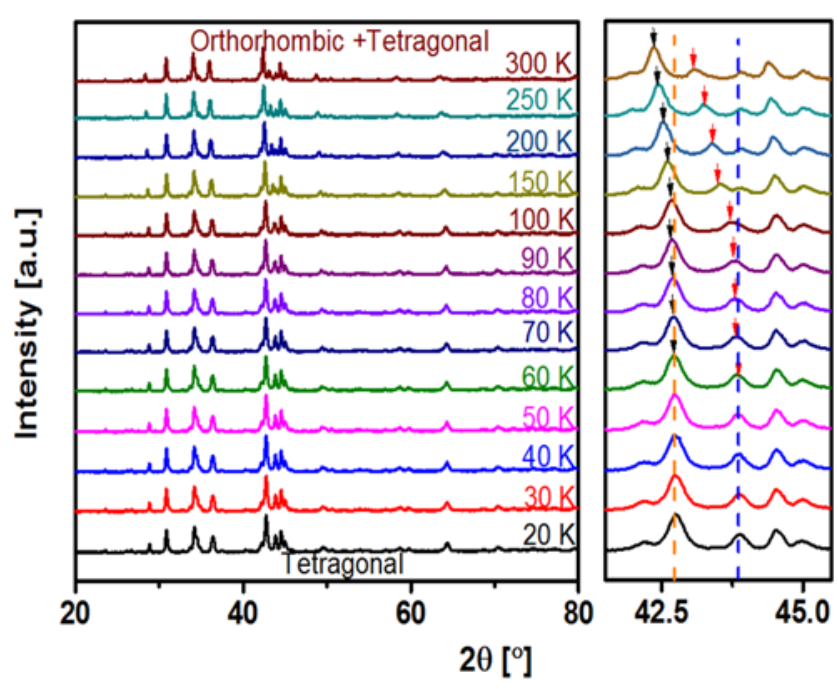

Figure 5. Temperature dependent XRD result for the sintered CuAgSe pallet from $20 \mathrm{~K}$ to $300 \mathrm{~K}$.

bulk $\mathrm{CuAgSe}^{18}$ [black dotted line in Figure $\left.4(\mathrm{f})\right]$, the pellet made from nanoparticles exhibits a higher $Z T$ due to extremely low thermal conductivity.

To investigate the abrupt metallic- $n$ transition at around 6o K, temperature-dependent XRD of pellet was performed in the range of $20 \mathrm{~K}$ to $300 \mathrm{~K}$ (Figure 5 ). As labeled by black and red arrows in Figure 5, with the temperature decreasing from $300 \mathrm{~K}$ to $60 \mathrm{~K}$, the strongest peak (152) orth shifted to right. Meanwhile, the two peaks of (003) tetra and (200) tetra gradually merged into one at around $70-60 \mathrm{~K}$. The sample remained the same crystal structure below 6o K. The dependence of lattice parameters on temperature was calculated and shown in Table S1. Apparently, both the lattice parameters and the volume of unit cell reduced with decrease of temperature.

The shrinkage of unit volume with temperature dropping is understandable. As illustrated previously, the tetragonal and orthorhombic CuAgSe are almost the same except that the later one has a sequence which repeats itself periodically every five tetragonal cells in the $b$ direction. The relative positions of atoms could be rearranged with temperature, leading to the structure transition from orthorhombic to tetragonal phase. This phase transition makes the structure more symmetric because the unit cell changes from bigger orthorhombic cell to smaller tetragonal one, which benefits to the reduction of scatterings of phonons and electrons. The gradually reduced electron scattering led to a high mobility, while reduced phonon scattering led to the increase in thermal conductivity with temperature decreasing to $22 \mathrm{~K}$ [Figure $4(\mathrm{e})]$.

The low temperature transport properties of $\mathrm{CuAgSe}$ were characterized by Hall Effect measurements. Detailed device assembly, the measured relationship between the Hall resistivity and the magnetic field, the calculated carrier density $(n)$, and the carrier mobility $(\mu)$ are presented in Figure 6(ad). The detailed calculations and results are presented in the Supporting Information (Section 3 and Table S2). The results show that electrons are the main charge carriers in $\mathrm{CuAgSe}$
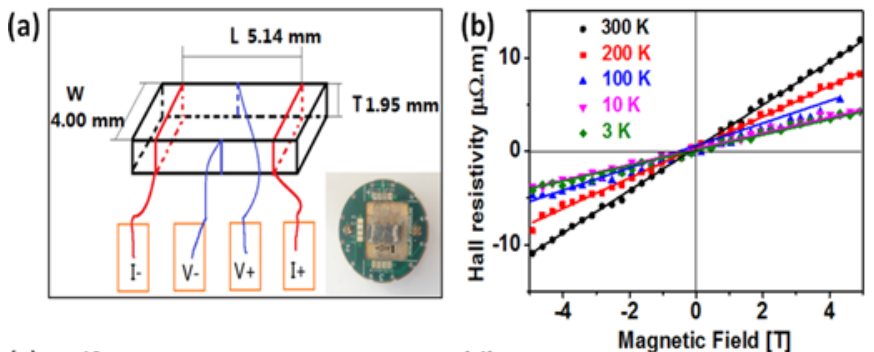

(c)
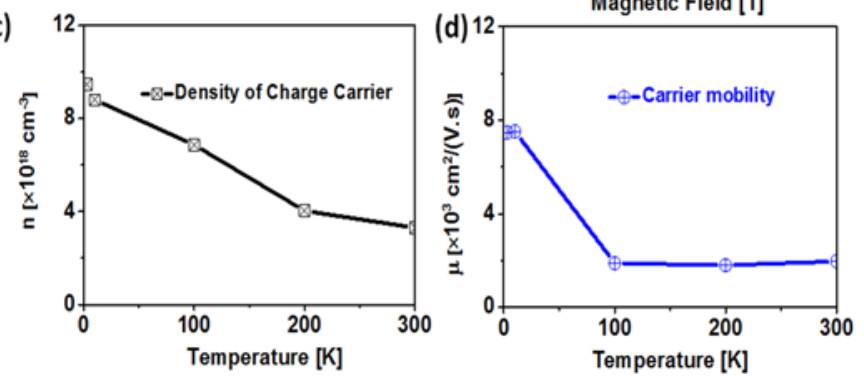

Figure 6. (a) Sample connections and dimensions for the measurement, inset is the assembled device for testing; (b) Hall resistivity as a function of magnetic field; (c) calculated $n$ and (d) calculated $\mu$ as functions of temperature. 
below $300 \mathrm{~K}$. The carrier density $(n)$ is approximately $10^{18}$ and decreases with rising temperature, while the carrier mobility first decreases from 7478 to $1798 \mathrm{~cm}^{2} /(\mathrm{V} \cdot \mathrm{s})$ with increasing temperature from $3 \mathrm{~K}$ to $200 \mathrm{~K}$, then slightly increases to $1966 \mathrm{~cm}^{2} /(\mathrm{V} \cdot \mathrm{s})$ at $300 \mathrm{~K}$. The carrier density is almost the same but the carrier mobility is significantly reduced by nanostructure compared with bulk CuAgSe. ${ }^{18-19}$

The thermoelectric properties of sintered $\mathrm{CuAgSe}$ nanoparticles above room temperature were further investigated, and Figure 7 displays the results measured from room temperature to $623 \mathrm{~K}$. The differences between the results around room temperature shown in Figures 4 and 7 originated from the error of different instruments. A reversible first order phase transition is proved by the heating-cooling DSC curve displayed in Figure $\mathrm{S}_{4}(\mathrm{a})$. This means the occurrence of a reversible phase transition during the heating process (i.e. changing from orthorhombic $\mathrm{CuAgSe}$ into cubic $\mathrm{CuAgSe}$ ), which has been demonstrated in previous reports. ${ }^{27-28}$ Like other $\mathrm{Cu}$ and Ag based PLEC materials with phase transition, ${ }^{16-17}$ the temperature dependences of the electrical conductivity, Seebeck coefficient, thermal conductivity, and specific heat of $\mathrm{CuAgSe}$ show an interesting transition accompanied by its phase transition. As presented in Figure 1(c), the high-temperature cubic phase is composed of a facecentered-cubic Se lattice with $\mathrm{Ag} / \mathrm{Cu}$ cations that are randomly distributed among the tetrahedral sites. This unique structure ensures the high mobility of the $\mathrm{Ag} / \mathrm{Cu}$ cations and leads to superionic property. The electrical conductivity of CuAgSe [Figure 7(a)] decreases from $1040 \mathrm{~S} / \mathrm{cm}$ to $400 \mathrm{~S} / \mathrm{cm}$ with increasing temperature from $323 \mathrm{~K}$ to $467 \mathrm{~K}$. After the phase transition, the scattering of charge carriers is dramatically increased by the highly disordered $\mathrm{Ag}^{+}$and $\mathrm{Cu}^{+}$ions, resulting in a further decrease in the electrical conductivity to $100 \mathrm{~S} / \mathrm{cm}$, which remains unchanged up to $623 \mathrm{~K}$. The independence of the electrical conductivity from temperature is related with the band structure of $p$-type CuAgSe, which is shown in Figure 8 and discussed late.
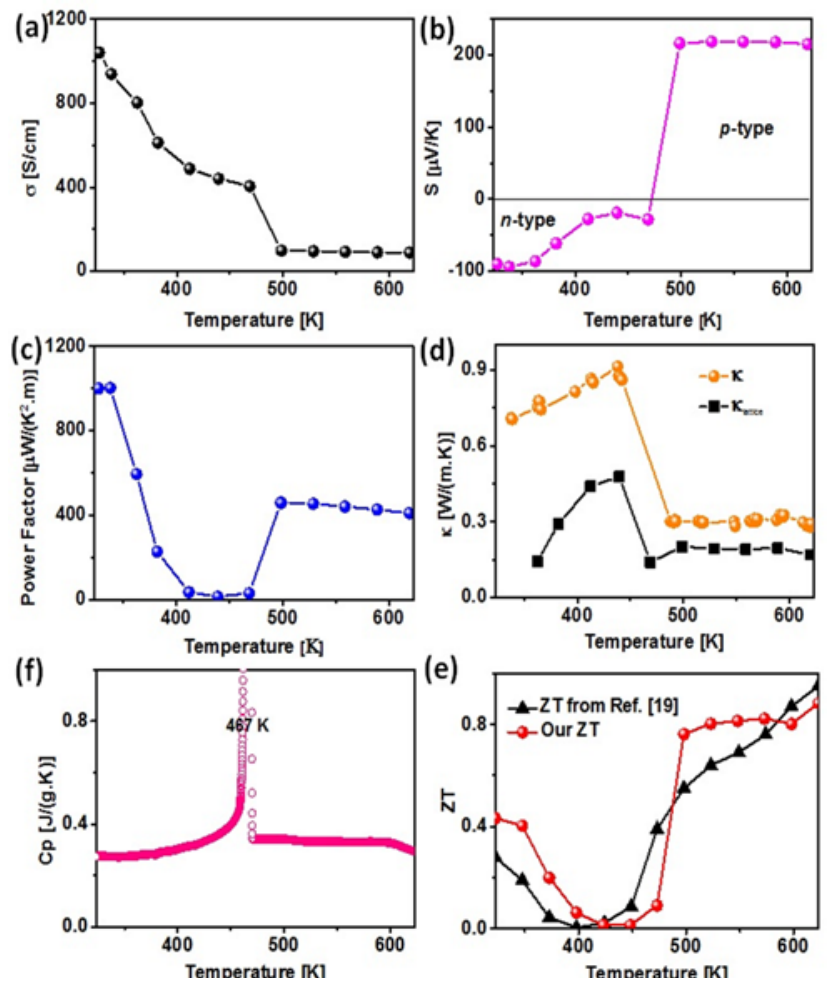

Figure 7. TE performance of sintered CuAgSe nanoparticles from $323 \mathrm{~K}$ to $623 \mathrm{~K}$ : (a) electrical conductivity; (b) Seebeck coefficient; (c) power factor; (d) thermal conductivity; (e) specific heat capacity; (f) ZT value.
The negative value of the Seebeck coefficient [Figure 7 (b)] demonstrates that orthorhombic $\mathrm{CuAgSe}$ is an intrinsic ntype semiconductor before the phase transition. The Seebeck coefficient decreases from $-90 \mu \mathrm{V} / \mathrm{K}$ to around $-20 \mu \mathrm{V} / \mathrm{K}$ with increasing temperature from room temperature to the phase transition temperature. A sudden $n-p$ transition occurs with the phase transition and the cubic $\mathrm{CuAgSe}$ exhibits a nearly constant positive Seebeck coefficient of $226 \mu \mathrm{V} / \mathrm{K}$ [Figure 7 (b)]. The transition of Seebeck coefficient (or type of charge carrier) is essentially due to the change of band structure, which is associated with the change of crystal structure arising from the variation of temperature. Increase of temperature leads to the transition from mixed orthorhombic and tetragonal phases into cubic structure, which has been reported in the bulk CuAgSe. ${ }^{19}$

The overall and lattice thermal conductivities ( $\kappa_{\text {lattice }}=\kappa-$ $\left.L \sigma T, L=2.1 \times 10^{-8}(\mathrm{~W} \cdot \Omega) / \mathrm{K}^{2}\right)$, Wiedemann Franz Law) of $\mathrm{CuAgSe}$ in the range of $323 \mathrm{~K}$ to $623 \mathrm{~K}$ are presented in Figure $7(\mathrm{~d})$. The overall thermal conductivity increases from 0.75 $\mathrm{W} /(\mathrm{m} \cdot \mathrm{K})$ at $370 \mathrm{~K}$ to around $0.92 \mathrm{~W} /(\mathrm{m} \cdot \mathrm{K})$ at $445 \mathrm{~K}$, accompanied by increasing lattice thermal conductivity ( $\left.\kappa_{\text {lattice }}\right)$ from $0.15 \mathrm{~W} /(\mathrm{m} \cdot \mathrm{K})$ at $370 \mathrm{~K}$ to $0.5 \mathrm{~W} /(\mathrm{m} \cdot \mathrm{K})$ at $445 \mathrm{~K}$. After the phase transition, the overall thermal conductivity is reduced to around $0.3 \mathrm{~W} /(\mathrm{m} \cdot \mathrm{K})$, owing to the high mobility of $\mathrm{Cu}^{+}$and $\mathrm{Ag}^{+}$cations. The lattice thermal conductivity slightly increases to $0.2 \mathrm{~W} /(\mathrm{m} \cdot \mathrm{K})$, as the contribution of charge carriers is inhibited by the low electrical conductivity. The low thermal conductivity of pellet made from nanoparticles is further addressed by comparing with that of pellet sintered from bulk counterpart (Figure $\mathrm{S}_{5}$ ). Bulk CuAgSe was prepared by annealing CuAgSe nanoparticles at $600{ }^{\circ} \mathrm{C}$ for $24 \mathrm{~h}$ in a sealed quartz tube under vacuum, which was ground into powder [Figure $\mathrm{S}_{5}(\mathrm{a})$ ] and sintered by SPS at $430^{\circ} \mathrm{C}$ for 10 min under 6o MPa. The cross section shows high density and absence of nanostructures in the pellet [Figure $\mathrm{S}_{5}(\mathrm{~b})$ ]. The X-ray diffraction pattern is same to that of original $\mathrm{CuAgSe}$ nanopowder [Figure $\mathrm{S}_{5}(\mathrm{c})$ ]. The thermal conductivity of the pellet sintered from annealed $\mathrm{CuAgSe}$ is higher than that of pellet from nanopowder, which supports the presence of nanostructures can effectively decrease the thermal conductivity.

The temperature-dependent ZT of our CuAgSe nanoparticles is also compared with literature values from bulk $\mathrm{CuAgSe}^{19}$ in Figure $7(\mathrm{f})$. A similar temperature dependence is observed in both nanostructured and bulk CuAgSe, ${ }^{19}$ although our room-temperature ZT is higher than previous reports (i.e. 0.42 vs. 0.15 at $323 \mathrm{~K}$ from PPMS; 0.42 vs. 0.28 at $323 \mathrm{~K}$ from high-temperature instruments). In the range of 500-575 K, our ZT values ( 0.8) are also higher than the liter-
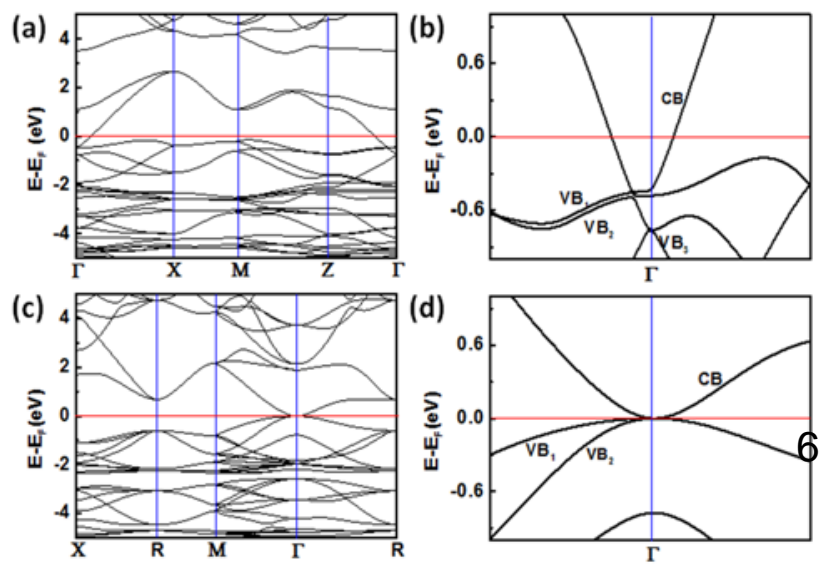

Figure 8. Calculated band structure for (a) tetragonal CuAgSe; (b) $\alpha$-phase cubic CuAgSe; and (c-d) magnified part of $\Gamma$ moint 
ature values. ${ }^{19}$ Our $Z T$ values above $575 \mathrm{~K}$ are comparable to the bulk value ( 0.9 vs. 0.95 at $623 \mathrm{~K}$ ) due to the extremely low thermal conductivity. The valley-like $Z T$ profiles suggest the minimum value near the phase transition temperature $(467 \mathrm{~K})$, and it drastically increased from 0.1 at $470 \mathrm{~K}$ to 0.8 at $500 \mathrm{~K}$. This anomalous behavior is consistent with previous reports on thermoelectric materials with phase transition. ${ }^{16-17}$

To further understand the $n-p$ transition of electrical conductivity, the electronic structures of tetragonal and cubic $\mathrm{CuAgSe}$ were calculated. Figure 8(a) illustrates the calculated band structure of tetragonal CuAgSe at the PBE level. Figure $8(\mathrm{~b})$ is the magnified band structure at $\Gamma$ point near the Fermi level. They both clearly show that one of conduction bands is crossing the Fermi level along the $\mathrm{Z}-\Gamma$ and $\Gamma-\mathrm{X}$ lines, while the top of valence band is just located below the Fermi level. ${ }^{18}$ Thus the tetragonal $\mathrm{CuAgSe}$ should possess two kinds of charge carriers. The main charge carriers are electrons, however, and the conductivity is $n$-type. In addition, the conduction bands are relative sparse than valence bands, and more electrons are located near Fermi level as shown by Figure 8(a) and its density of states (DOS) [Figure S6(a)]. Figure 8(b) also demonstrates that both the conduction bands and valence bands are highly asymmetric at the $\Gamma$ point, which means the tetragonal structure is highly anhomonic or disordered, leading to low thermal conductivity. Figure 8(c) is the calculated band structure of cubic $\mathrm{CuAgSe}$ and Figure 8(d) is the magnified band structure at $\Gamma$ point where no band gap was observed.

The Seebeck coefficient could be expressed by the following Equation (5):

$$
S=A \times T \times \frac{m^{*}}{n^{2 / 3}} \quad(5)
$$

In which $\mathrm{A}$ is a constant, $\mathrm{T}$ is temperature, $\mathrm{n}$ is the charge carrier density, $\mathrm{m}^{*}$ is the effective mass of charge carriers and equal to the reciprocal of the curvature of band edge when the band gap is o eV at $\Gamma$ point. As displayed in Figure 8(d), the curvature of conduction band edge $(\mathrm{CB})$ is bigger than any of the valence band edge $\left(\mathrm{VB}_{1}\right.$ and $\left.\mathrm{VB}_{2}\right)$, the effective mass of holes $\left(m_{h}^{*}=m_{V B 1}^{*}+m_{V B 2}^{*}\right)$ is heavier than the absolute value of electrons $\left(\left|m_{e}^{*}\right|\right)$. The whole effective mass is positive, e.g. $m^{*}=m_{e}^{*}+m_{h}^{*}>0$, which results in a positive $S$ [Figure $7(\mathrm{~b})]$. In addition, the high-temperature phase $\mathrm{CuAgSe}$ possesses a cubic-face-centered Se frame, but both the conduction band and valence band at $\Gamma$ point show high asymmetry [Figure $8(\mathrm{~d})$ ]. This implies the cations sublattice $\left(\mathrm{Cu}^{+}\right.$and $\left.\mathrm{Ag}^{+}\right)$is highly disordered, which can enhance the phonon scatterings and reduce the thermal conductivity. Although the DOS of cubic structure shows the same concentration near Fermi level [Figure S6(b)] as tetragonal phase, the electrical conductivity is still low due to the disordered cations. It has known that the electrical conductivity $(\sigma)$ of an intrinsic semiconductor can be expressed by Equation (6), ${ }^{16}$ in which $f(T)$ is a function almost independent with temperature (T), $E_{g}$ is the band gap, $k_{B}$ is Boltzmann constant. Due to that the band gap of p-type $\mathrm{CuAgSe}$ is o eV [Figure $8(\mathrm{~d})$ ], the electrical conductivity is almost a constant with temperature increasing.

$$
\sigma=f(T) \times \exp \left(-\frac{E_{g}}{k_{B} T}\right)
$$

From the perspective of practical application, the cycling stability of thermoelectric materials is a very important parameter during long-term operation. Therefore, we tested
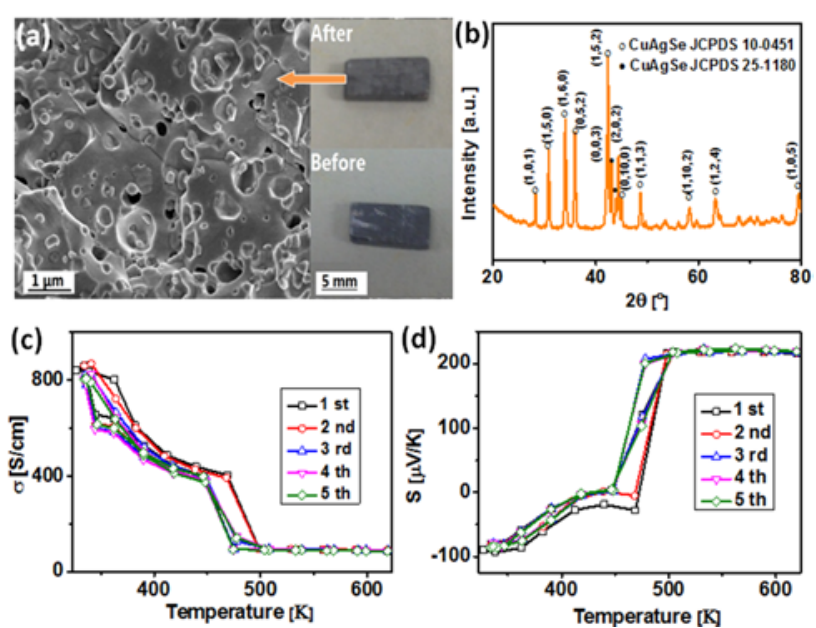

Figure 9. TE properties of sintered CuAgSe nanoparticles tested over 5 cycles (heating and cooling): (a) micromorphology after 5 cycles; right side is the macromorphology before and after 5 cycles; (b) XRD pattern after 5 cycles; (c) electrical conductivity; (d) Seebeck coefficient.

the stability of the consolidated CuAgSe pellet in a sealed chamber (Ozawa 2ooli) filled with a low pressure helium, and measured its electrical conductivity and Seebeck coefficient through 5 cycles of heating and cooling from room temperature to $623 \mathrm{~K}$. There are no big differences in the morphology, composition, and appearance of the pellet before and after 5 cycles. The morphology, composition, electrical conductivity, and Seebeck coefficient are plotted in Figure 9 (a-d). Good reproducibility is observed in these data obtained from different cycles. The heating-cooling cycles demonstrate the good stability of the pellet, which is also supported by the zero loss in weight during the thermogravimetric analysis (TGA) [Figure $\mathrm{S}_{4}(\mathrm{~b})$ ]. No oxidation or evaporation occurred during analysis. In addition, XPS was also employed to characterize the sample after 5 cycles (Figure $\mathrm{S}_{7}$ ). No other elements were detected, and the valence states of $\mathrm{Cu}$, Se and $\mathrm{Ag}$ did not change. The same results were obtained before and after 5 cycles of tests demonstrate that our $\mathrm{CuAgSe}$ pellet consolidated from nanoparticles is stable under cycling.

\section{CONCLUSION}

Large-scale surfactant-free CuAgSe nanoparticles were successfully synthesized via a robust environmentally friendly aqueous method. The resultant $\mathrm{CuAgSe}$ nanoparticles were sintered into pellets by an advanced SPS technique for thermoelectric investigation in the range of $3 \mathrm{~K}$ to $623 \mathrm{~K}$. The results show an interesting temperature-dependent transport property accompanied by a phase transition, i.e. sintered $\mathrm{CuAgSe}$ nanoparticles exhibit metallic characteristics below 6o $\mathrm{K}, n$-type semiconductor behavior in the range of $60 \mathrm{~K}$ to $480 \mathrm{~K}$, and $p$-type semiconducting behavior above $480 \mathrm{~K}$. The pellet displays a ZT of 0.42 at $323 \mathrm{~K}$ and 0.9 at $623 \mathrm{~K}$, and exhibits excellent cycling stability. The temperaturedependent $n-p$ transition and the excellent stability of $\mathrm{CuAgSe}$ enable it to simultaneously serve as an $n$-type and $p$ type (at different temperatures) thermoelectric candidate for conversion of heat into electricity.

\section{ASSOCIATED CONTENT}




\section{Supporting Information}

Electronic Supplementary Information (ESI) available: results of control experiment, including XRD patterns and SEM images, and DSC, TGA, DOS and XPS curves of the CuAgSe pellet, calculated lattice parameters and transport properties, as well as the Hall Effect calculations are included in the Supporting Information. This material is available free of charge via the Internet at http://pubs.acs.org.

\section{AUTHOR INFORMATION}

\section{Corresponding Author}

*Dr. Z. Li (zhenl@uow.edu.au); Fax: 61-2-42215731; Tel: 61-242215163 .

\section{Author Contributions}

All authors have given approval to the final version of the manuscript.

\section{ACKNOWLEDGMENTS}

C. Han gratefully acknowledges the Chinese Scholarship Council (CSC) for his scholarship. Z. Li and M. Lu acknowledge support from the Australian Research Council (ARC) through the Discovery Projects DP130102699 and DP130102274. S. Dou is grateful for support from the Baosteel-Australia Research Centre (BARC) through the project BA11oon and from the ARC through the Linkage Project LP120200289. The authors would like to thank Dr. Tania Silver for polishing the manuscript, and ISEM and the Electronic Microscope Centre (EMC) for their support.

\section{REFERENCES}

(1) Bell, E. Science. 2008, 321, 1457-1461.

(2) Xiao, C.; Xu, J.; Li, K.; Fen, J.;Yang, J. L.; Xie, Y. J. Am. Chem. Soc. 2012, 134, 4287-4293.

(3) Kraemer, D.; Poudel, B.; Feng, H. P.; Caylor, J. C.; Bo, Y.; Yan, X.; Ma, Y.; Wang, X. W.; Wang, D. Z.; Muto, A.; McEnaney, K.; Chiesa, M.; Ren, Z. F.; Chen, G. Nat. Mater. 2011, 10, 532-538.

(4) Liu, W. S.; Zhang, Q. Y.; Lan, Y. C.; Chen, S.; Yan, X.; Zhang, Q.; Wang, H.; Wang, D. Z.; Chen, G.; Ren, Z. F. Adv. Energy Mater. 2o11, $1,577-587$.

(5) Hoshino, A.; Fujioka, K.; Oku, T.; Nakamura, S.; Suga, M.; Yamaguchi, Y.; Suzuki, K.; Yasuhara, M.; Yamamoto, K. Immunol. 20o4, 48, 985-994.

(6) Pichanusakorn, P.; Bandaru, P. Mater. Sci. E Eng. R. 2o1o, 67, 1963.
(7) Shakouri, A. Annu. Rev. Mater. Res. 2011, 41, 399-431.

(8) Snyder, G. J.; Toberer, E. S. Nat. Mater. 2008, 7, 105-114.

(9) Li, Z.; Sun, Q.; Yao, X. D.; Zhu, Z. H.; Lu, G. Q. J. Mater. Chem. 2012, 22, 22821-22831.

(10) Han, C.; Li, Z.; Dou, S. Chin. Sci. Bull. 2014, 59, 2073-2091.

(11) Han, C.; Li, Z.; Li, W. J.; Chou, S. L.; Dou, S. J. Mater. Chem. A. 2014, 2, 11683-1169o.

(12) Liu, H. L.; Shi, X.; Xu, F. F.; Zhang, L. L.; Zhang, W. Q.; Chen, L. D.; Li, Q.; Uher, C.; Day, T.; Snyder, G. J. Nat. Mater. 2012, 11, 422425

(13) Slack, G. A. Solid. State. Phys. 1979, 34, 1-71.

(14) Kanishka, B.; He, J.; Ivan, D. B.; Wu, C.; Hogan, T. P.; Seidman, D. N.; Dravid, V. P.; Kanatzidis, M. G. Nature. 2012, 489, 414-418.

(15) He, Y.; D, T.; Zhang, T. S.; Liu, H. L.; Shi, X.; Chen, L. D.; Snyder, G. J. Adv. Mater. 2014, 26, 3974-3978.

(16) Mi, W. L.; Qiu, P. F.; Zhang, T. S.; Lv, Y. H.; Shi, X.; Chen, L. D. Appl. Phys. Lett. 2014, 104, 133903(1-5).

(17) Liu, H. L.; Yan, X.; Lu, P.; Shi, X.; Xu, F. F.; He, Y.; Tang, Y. S.; Bai, S. Q.; Zhang, W. Q.; Chen, L. D.; Lin, Y.; Shi, L.; Lin, H.; Gao, X. Y.; Zhang, X. M.; Chi, H.; Uher, C. Adv. Mater. 2013, 25, 6607-6612.

(18) Ishiwata, S.; Shiomi, Y.; Lee, J. S.; Bahramy, M. S.; Suzuki, T.; Uchida, M.; Arita, R.; Taguchi, Y.; Tokura, Y. Nat. Mater. 2013, 12, 512517 .

(19) Hong, A. J.; Li, L.; Zhu, H. X.; Zhou, X. Y.; He, Q. Y.; Liu, W. S.; Yan, Z. B.; Liu, J. M.; Ren, Z. F. Solid. State. Ionics. 2014, 261, 21-25.

(20) Fang, C. X.; Zhang, S.; Zuo, P. F.; Wei, W.; Jin, B. K.; Wu, J. Y.; Tian, Y. P. J. Cryst. Growth. 2009, 311, 2345-2351.

(21) Gao, Y. H.; Zheng, Z., Tian, Y. P.; Zhang, Y. D.; Zhang, Y. Eur. J. Inorg. Chem. 2011, 27, 4198-4203.

(22) Perdew, J. P.; Burke, K.; Ernzerhof, M. Phys. Rev. Lett. 1996, 77, 3865-3868.

(23) Delley, B. J. Chem. Phys. 1990, 92, 508-517.

(24) Delley, B. J. Chem. Phys. 2ooo, 113, 7756-7764.

(25) Sun, Q.; Li, Z.; Searles, D. J.; Chen, Y.; Lu, G. Q.; Du, A. J. J. Am. Chem. Soc. 2013, 135, 8246-8253.

(26) Frueh, A. J.; Czamanske, G. K.; Knight, C. H. Zeit. Krist. 1957, 108, 389-396.

(27) Bikkulova, N.N.;Assylguzhina, G.N.;Skomorokhov, A.N.; Yadrovskii, E.L.; Beskrovnyi, A. I.; Stepanov, Y.M. Bull. Russ. Acad. Sci: Phys. 20o6, 7o, 641-643.

(28) Baikulov, R. B.; Asadov, Y. G. Inorg. Mater. 2005, 41, 407-411.

(29) Chen,X. Q.; Li, Z.; Bai,Y.; Sun Q.; Wang, L. Z.; Dou, S. X. Chem. Eur. J. 2014, 20, 1-10.

(30) Xiong, J. Y.; Li, Z.; Chen, J.; Zhang, S. Q.; Wang, L. Z.; Dou, S. ACS Appl. Mater. Interfaces. 2014, 6, 15712-15722.

(31) Vanderputten, D.; Ohta, D.; Zanoni, R. Mater. Sci. Forum. 1995, 195, 123-126.

(32) Riha, S. C.; Johnson, D. C.; Prieto, A. L. J. Am. Chem. Soc. 2011, 133, 1383-1390.

(33) Domashevskaya, E. P.; Gorbachev, V. A.; Terekhov, V. A.; Kashkarov, V. M.; Panfilova, E. V.; Shchukarev, A. V. J. Electron. Spectrosc. Relat. Phenom. 2001, 114, 901-908. 


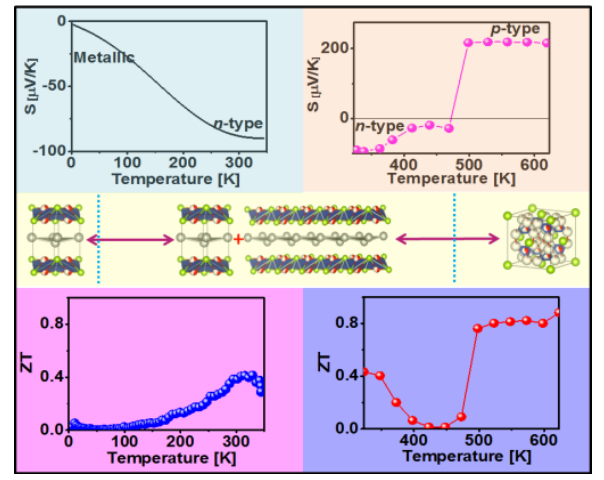

